\title{
Radiation Effect on Mixed Convection Flow of a Micropolar Fluid over an Unsteady Stretching Surface with Viscous Dissipation and Heat Generation
}

\author{
M. Suryanarayana Reddy ${ }^{1}$ and S. Vijaya Lakshmi ${ }^{2}$ \\ ${ }^{l}$ Department of mathematics, JNTUA College of engineering, Pulivendula-516 390.A.P, India \\ ${ }^{2}$ Research Scholar, JNTU Anantapur, Anantapuramu, A.P, India.
}

\begin{abstract}
The unsteady, two dimensional, mixed convection flow of an viscous incompressible electrically conducting micropolar fluid over a vertical and impermeable stretching surface in the presence of Heat source/sink and radiation when the buoyancy force assists and opposes the flow has been studied. Using the similarity transformations, the governing equations have been transformed into a system of ordinary differential equations. These differential equations are highly nonlinear which cannot be solved analytically. Therefore, forth order Runge-Kutta method along with shooting technique has been used for solving it. Numerical results are obtained for the skin-friction coefficient, the couple wall stress and the local Nusselt number as well as the velocity, microrotation and temperature profiles for different values of the governing parameters, namely, material parameter, unsteadiness parameter, heat source/sink parameter, radiation parameter and Eckert number.
\end{abstract}

Keywords: unsteady flow, mixed convection, Heat Transfer, micropolar fluid, Stretching surface, heat generation, radiation and viscous dissipation.

\section{Introduction}

The theory of micropolar fluids has received great attention during the recent years, because the traditional Newtonian fluids cannot precisely describe the characteristic of fluid with suspended particles. A micropolar fluid obeys the constitutive equations of the considered non-Newtonian fluid model. In the micropolar fluid model, apart from the classical velocity field, a microrotation vector and a gyration parameter are introduced in order to investigate the kinematics of microrotation. Such fluid model may be applied to explain the flow of colloidal solutions, liquid crystals, fluids with additives, suspension solutions, animal blood, etc. The presence of dust or smoke particular in a gas may also be modeled using micropolar fluid dynamics. Unlike the other fluids, micropolar fluids are fluids with microstructure belonging to a class of fluids with nonsymmetrical stress tensor. Physically, they represent fluids consisting of randomly oriented particles suspended in a viscous medium. The theory of micropolar fluids, first proposed by Eringen $[1,2]$ is capable of describing such fluids. In this theory the local effects arising from the microstructure and the intrinsic motion of the fluid elements are taken into account. This is a kind of continuum mechanics, and many classical flows are being reexamined to determine the effects of fluid microstructure [3-5]. Early studies along these lines may be found in the review article by Peddieson and McNitt [6], and in the recent books by Lukaszewicz [7] and Eringen [8]. The boundary layer flow and heat transfer in a quiescent Newtonian and non- Newtonian fluid driven by a continuous stretching sheet is of significance in a number of industrial engineering processes, such as the drawing of a polymer sheet or filaments extruded continuously from a die, the cooling of a metallic plate in a bath, the aerodynamic extrusion of plastic sheets, the continuous casting, rolling, annealing and tinning of copper wires, the wire and fiber coating, etc. During the processes, mechanical properties are greatly dependent upon the rate of cooling.

The heat source/sink effects in thermal convection, are significant where there may exist a high temperature differences between the surface (e.g. space craft body) and the ambient fluid. Heat generation is also important in the context of exothermic or endothermic chemical reactions. Postelnicu et al. [9] investigated the effect of variable viscosity on forced convection over a horizontal flat plate in a porous medium with internal heat generation. Molla et al. [10] studied natural convection flow along a vertical wavy surface with uniform surface temperature in presence of heat generation/absorption. MHD heat and mass transfer free convection flow along a vertical stretching sheet in presence of magnetic field with heat generation are studied by Samad et al. [11]. Alam et al [12] analyzed the study of the combined free - forced convection and mass transfer flow past a vertical porous plate in a porous medium with heat generation and thermal diffusion. Recently, Rahman et al. [13] investigated the thermophoresis effect on MHD forced convection on a fluid over a continuous linear stretching sheet in presence of heat generation and Power-Law wall temperature 
Dissipation is the process of converting mechanical energy of downward-flowing water into thermal and acoustical energy. Various devices are designed in streambeds to reduce the kinetic energy of flowing waters, reducing their erosive potential on banks and river bottoms. Vajravelu and Hadjinicalaou [14] analyzed the heat transfer characteristics over a stretching surface with viscous dissipation in the presence of internal heat generation or absorption. Gebhart [15] has shown the importance of viscous dissipative heat in free convection flow in the case of isothermal and constant heat flux at the plate. Gebhart and Mollendorf [16] considered the effects of viscous dissipation for external natural convection flow over a surface. Recently, Abd El-Aziz [17] studied the mixed convection flow of a micropolar fluid from an unsteady stretching surface with viscous dissipation.

Ghaly [18] considered the thermal radiation effect on a steady flow, whereas Rapits and Massalas [19] and El-Aziz [20] analyzed the unsteady case. Sattar and Alam [21] presented unsteady free convection and mass transfer flow of a viscous, incompressible, and electrically conducting fluid past a moving infinite vertical porous plate with thermal diffusion effect. $\mathrm{Na}$ and Pop [22] analyzed an unsteady flow due to a stretching sheet. In the case of unsteady boundary-layer flow, Singh et al. [23] investigated the thermal radiation and magnetic field effects on an unsteady stretching permeable sheet in the presence of free stream velocity. Recently, Khan et al. [24] studied the unsteady MHD free convection boundary-layer flow of a nanofluid along a stretching sheet with thermal radiation and viscous dissipation effects.

The present study investigates the unsteady mixed convection flow of a viscous incompressible electrically conducting micropolar fluid on a vertical and impermeable stretching sheet in the presence of heat generation or absorption and radiation. Using the similarity transformations, the governing equations have been transformed into a set of ordinary differential equations, which are nonlinear and cannot be solved analytically, therefore, fourth order Runge-Kutta method along with shooting technique has been used for solving it. The results for velocity, microrotation and temperature functions are carried out for the wide range of important parameters namely, material parameter, radiation parameter, Eckert number, unsteadiness parameter and heat source/sink parameter. The skin friction, the couple wall stress and the rate of heat transfer have also been computed.

\section{Mathematical Formulation}

Consider an unsteady two dimensional, mixed convection boundary layer flow of a viscous incompressible and radiating micropolar fluid over an elastic, vertical and impermeable stretching sheet which emerges vertically in the upward direction from a narrow slot with velocity [22]

$$
U_{w}(x, t)=\frac{a x}{1-\alpha t}
$$

where both $a$ and $\alpha$ are positive constants with dimension per time. The positive $x$ coordinate is measured along the stretching sheet with the slot as the origin and the positive $y$ coordinate is measured normal to the sheet in the outward direction toward the fluid. The surface temperature $T_{w}$ of the stretching sheet varies with the distance $\mathrm{x}$ from the slot and time $t$ as

$$
T_{w}(x, t)=T_{\infty}+\frac{b x}{(1-\alpha t)^{2}}
$$

where $b$ is constant with dimension temperature and concentration over length and $v$ is the kinematic viscosity of the ambient fluid. It is apt to note here that, the expressions for $U_{w}(x, t)$ and $T_{w}(x, t)$ in Eqs. (2.1) and (2.2) are valid only for time $t=\alpha^{-1}$ unless $\alpha=0$. Expression (2.1) for the velocity of the sheet $U_{w}(x, t)$ reflects that the elastic sheet which is fixed at the origin is stretched by applying a force in the positive $x$ direction and the effective stretching rate $\frac{a}{(1-\alpha t)}$ increases with time. With the same analogy the expression for the surface temperature $T_{w}(x, t)$ is given by Eq. (2.2) represents a situation in which the sheet temperature increases (reduces) if $\mathrm{b}$ is positive (negative) from $T_{\infty}$ at the slot in proportion to $x$ and such that the amount of temperature increase (reduction) along the sheet increases with time. It is assumed that the Dufour effects are neglected in the energy equation. It is further assumed that the fluid properties are taken to be constant except for the density variation with the temperature in the buoyancy term. Under the usual boundary layer approximation, the governing equations are

Continuity equation

$$
\frac{\partial u}{\partial x}+\frac{\partial v}{\partial y}=0
$$

Linear momentum equation 


$$
\frac{\partial u}{\partial t}+u \frac{\partial u}{\partial x}+v \frac{\partial u}{\partial y}=\left(\frac{\mu+\kappa}{\rho}\right) \frac{\partial^{2} u}{\partial y^{2}}+\frac{\kappa}{\rho} \frac{\partial N}{\partial y}+g \beta\left(T-T_{\infty}\right)
$$

Momentum equation

$$
\rho j\left(\frac{\partial N}{\partial t}+u \frac{\partial N}{\partial x}+v \frac{\partial N}{\partial y}\right)=\gamma \frac{\partial^{2} N}{\partial y^{2}}-\kappa\left(2 N+\frac{\partial u}{\partial y}\right)
$$

Energy equation

$$
\frac{\partial T}{\partial t}+u \frac{\partial T}{\partial x}+v \frac{\partial T}{\partial y}=\alpha_{0} \frac{\partial^{2} T}{\partial y^{2}}-\frac{1}{\rho c_{p}} \frac{\partial q_{r}}{\partial y}+\left(\frac{\mu+\kappa}{\rho c_{p}}\right)\left(\frac{\partial u}{\partial y}\right)^{2}+Q_{0}\left(T-T_{\infty}\right)
$$

The boundary conditions for the velocity, angular velocity and temperature fields are

$$
\begin{aligned}
& u=U_{w}, v=0, N=0, T=T_{w} \quad \text { at } \quad y=0 \\
& u \rightarrow 0, N \rightarrow 0, T \rightarrow T_{\infty} \quad \text { as } \quad y \rightarrow \infty
\end{aligned}
$$

Where $u$ and $v$ are the velocity components in the $x$ - and $y$ - directions, respectively, $T$ is the fluid temperature in the boundary layer, $N$ is the component of the microrotation vector normal to the x-y plane, $\sigma$ is the spin-gradient viscosity and $\alpha_{0}\left(=k / \rho c_{p}\right)$ is the thermal diffusivity with $k$ is the fluid thermal conductivity, $q_{r}$ is the heat flux, $Q_{0}$ is the heat generation coefficient, $c_{p}$ is the heat capacity pressure, respectively.

By using the Rosseland approximation the radiative heat flux $q_{r}$ is given by

$$
q_{r}=-\frac{4 \sigma_{1}}{3 k^{*}} \frac{\partial T^{4}}{\partial y}
$$

Where $\sigma_{1}$ is the Stefan -Boltzmann constant and $k *$ is the mean absorption coefficient. It should be noted that by using the Rosseland approximation, the present analysis is limited to optically thick fluids. If temperature differences within the flow are significantly small, then equation (2.6) can be linearised by expanding $T^{4}$ into the Taylor series about $T_{\infty}$, which after neglect higher order terms takes the form:

$$
T^{4} \cong 4 T_{\infty}^{3} T-3 T_{\infty}^{4}
$$

In view of equations (2.8) and (2.9), eqn. (2.6) reduces to

$$
\frac{\partial T}{\partial t}+u \frac{\partial T}{\partial x}+v \frac{\partial T}{\partial y}=\left(\alpha_{0}+\frac{16 \sigma_{1} T_{\infty}^{3}}{3 k^{*} \rho c_{p}}\right) \frac{\partial^{2} T}{\partial y^{2}}+\left(\frac{\mu+\kappa}{\rho c_{p}}\right)\left(\frac{\partial u}{\partial y}\right)^{2}+Q_{0}\left(T-T_{\infty}\right)
$$

The continuity equation (2.3) is satisfied by the Cauchy Riemann equations

$$
u=\frac{\partial \psi}{\partial y} \text { and } v=-\frac{\partial \psi}{\partial x}
$$

where $\psi(x, y)$ is the stream function.

In order to transform equations (2.4), (2.5) and (2.10) into a set of ordinary differential equations, the following similarity transformations and dimensionless variables are introduced.

$$
\begin{aligned}
& \eta=\sqrt{\frac{a}{v(1-\alpha t)}} y, \psi=\sqrt{\frac{v a}{1-\alpha t}} x f(\eta), N=\sqrt{\frac{a^{3}}{v(1-\alpha t)^{3}}} x h(\eta) \\
& T=T_{\infty}+\frac{b x}{(1-\alpha t)^{2}} \theta(\eta), A=\frac{\alpha}{a}, K=\frac{\kappa}{\mu}, \operatorname{Pr}=\frac{v}{\alpha_{0}}, \lambda_{0}=\frac{\gamma}{\mu j} \\
& G r_{x}=\frac{g \beta\left(T_{w}-T_{\infty}\right) x^{3}}{v^{2}}, \operatorname{Re}_{x}=\frac{U_{w} x}{v}, \xi=\frac{g \beta b}{a^{2}}=\frac{G r_{x}}{\operatorname{Re}_{x}^{2}} \\
& B=\frac{v(1-\alpha t)}{j b}=\frac{v x}{j U_{w}}, E c=\frac{U_{w}^{2}}{c_{p}\left(T_{w}-T_{\infty}\right)}, Q=\frac{Q_{0}}{\rho c_{p} a}, R=\frac{16 \sigma_{1} T_{\infty}^{3}}{3 k * \rho c_{p}}
\end{aligned}
$$

where $f(\eta)$ is the dimensionless stream function, $\theta$ is the dimensionless temperature, $\eta$ is the similarity variable, $A$ is the unsteadiness parameter, $E c$ is the Eckert number, $Q$ is the heat source parameter, $G r_{x}$ is the thermal Grashof number, $\xi$ is the thermal buoyancy parameter, $\lambda_{0}, B$ are the dimensionless 
parameters, $\operatorname{Re}_{x}$ is the local Reynolds number, $\mathrm{R}$ is the radiation parameter and $\operatorname{Pr}$ is the Prandtl number.

In view of equations (2.11) and (2.12), the equations (2.4), (2.5) and (2.10) transform into

$$
\begin{aligned}
& (1+K) f^{\prime \prime \prime}+f f^{\prime \prime}-\left(f^{\prime}\right)^{2}+K h^{\prime}-\frac{A}{2}\left(2 f^{\prime}+\eta f^{\prime \prime}\right)+\xi \theta=0 \\
& \lambda_{0} h^{\prime \prime}+f h^{\prime}-f^{\prime} h-K B\left(2 h+f^{\prime \prime}\right)-\frac{A}{2}\left(3 h+\eta h^{\prime}\right)=0 \\
& (1+R) \frac{1}{\operatorname{Pr}} \theta^{\prime \prime}+f \theta^{\prime}-f^{\prime} \theta-\frac{A}{2}\left(4 \theta+\eta \theta^{\prime}\right)+Q \theta+E c(1+K)\left(f^{\prime \prime}\right)^{2}=0
\end{aligned}
$$

The corresponding boundary conditions are

$$
\begin{aligned}
& f=0, f^{\prime}=1, h=0, \theta=1 \quad \text { at } \quad \eta=0 \\
& f^{\prime}=h=\theta=0 \quad \text { as } \quad \eta \rightarrow \infty
\end{aligned}
$$

where the primes denote differentiation with respect to $\eta$

The physical quantities of interest are the skin friction coefficient $C_{f x}$, the local couple wall stress $M_{w x}$ and the local Nusselt number $N u_{x}$ which are defined as

$$
\begin{aligned}
& C_{f x}=\frac{2}{\rho U_{w}^{2}}\left[(\mu+\kappa)\left(\frac{\partial u}{\partial y}\right)_{y=0}+\kappa(N)_{y=0}\right]=2(1+K) \operatorname{Re}_{x}^{-1 / 2} f^{\prime \prime}(0) \\
& M_{w x}=\gamma\left(\frac{\partial N}{\partial y}\right)_{y=0}=\frac{\gamma a U_{w}}{v(1-\alpha t)} h^{\prime}(0) \\
& N u_{x}=-\frac{x}{T_{w}-T_{\infty}}\left(\frac{\partial T}{\partial y}\right)_{y=0}=-\operatorname{Re}_{x}^{2} \theta^{\prime}(0)
\end{aligned}
$$

Our main aim is to investigate how the values of $f^{\prime \prime}(0), h^{\prime}(0)$ and $-\theta^{\prime}(0)$ vary in terms of the various parameters.

\section{Solution Of The Problem}

The set of coupled non-linear governing boundary layer equations (2.13) - (2.15) together with the boundary conditions (2.16) are solved numerically by using Runge-Kutta fourth order technique along with shooting method. First of all, higher order non-linear differential Equations (2.13) - (2.15) are converted into simultaneous linear differential equations of first order and they are further transformed into initial value problem by applying the shooting technique (Jain et al.[25]). The resultant initial value problem is solved by employing Runge-Kutta fourth order technique. The step size $\Delta \eta=0.05$ is used to obtain the numerical solution with five decimal place accuracy as the criterion of convergence. From the process of numerical computation, the skin-friction coefficient, wall couple stress and the Nusselt number which are respectively proportional to $f^{\prime \prime}(0), h^{\prime}(0)$ and $-\theta^{\prime}(0)$ are also sorted out and their numerical values are presented in a tabular form.

\section{Results And Discussion}

The governing equations (2.13) - (2.15) subject to the boundary conditions (2.16) are integrated as described in section 3. In order to get a clear insight of the physical problem, the velocity, angular velocity and temperature have been discussed by assigning numerical values to the parameters encountered in the problem.

Physically $\xi>0$ means heating of the fluid or cooling of the surface (assisting flow), $\xi<0$ means cooling of the fluid or heating of the surface (opposing flow) and $\xi=0$ means the absence of free convection currents (forced convection flow). Figs. 1-3 illustrate the axial velocity, angular velocity and temperature fields for different values of the buoyancy and unsteadiness parameters $\xi$ and $A$, respectively. For assisting flow, namely for positive value of $\xi$, it is seen from Fig. 1 that increasing of $\xi$ have tendency to induce more flow in the boundary layer for both values of $A$. For the opposing flows $(\xi<0)$, on the other hand, the effect of buoyancy is to reduce the velocity compared to those for pure forced convection $(\xi=0)$. This is due to the fact that a positive $\xi$ induces a favorable pressure gradient that enhances the fluid flow in the boundary layer, while a negative $\xi$ produces an adverse pressure gradient that slows down the fluid motion. Also, it is observed that for large values of the buoyancy parameter $(\xi=10)$ the velocity overshoots near the wall over the moving speed of the sheet. From Fig. 1 it is noticed that the least effect of $A$ on the axial velocity $f^{\prime}(\eta)$ occurs in the 
case of forced convection flow $(\xi=0)$ while greatest one occurs in the case of free convection flow $(\xi=10)$. Further, for $\xi=10$, the axial velocity $f^{\prime}(\eta)$ decreases as $A$ increases near the sheet where $0 \leq \eta \leq \eta_{0} \leq 2.7$ whereas for $\eta>\eta_{0}$, it rises slightly before it dies out at the end of the boundary layer. Moreover, the effect of $A$ on the axial velocity $f^{\prime}(\eta)$ in the case of opposing flow $(\xi<0)$ is insensible for $\eta \leq 1$ but $f^{\prime}(\eta)$ increases with an increase in the boundary layer thickness as $A$ increases for $\eta>1$.

For assisting flow, Fig. 2 shows for both values of $A$ that close to the sheet surface and at the last part of the boundary layer, an increase in the values of $\xi$ leads to the decrease in the values of the angular velocity $h(\eta)$ while for middle part of the boundary layer at a fixed $\eta$ position the angular velocity $h(\eta)$ increases with the increase of the buoyancy parameter $\xi$. For the opposing flows $\xi<0$, on the other hand, the angular velocity $h(\eta)$ increases greatly near the sheet where $0 \leq \eta<\eta_{0} \cong 1.6$ whereas for $\eta>\eta_{0}$, it reduces with a decrease in thickness of the angular momentum boundary layer. Also, for assisting flows, increasing values of the buoyancy parameter $\xi$ move the location of the maximum value of the angular velocity away from the surface while the opposite trend is noticed for opposing flow. From Fig 2 it is noteworthy that the direction of the angular velocity is negative very close to the sheet for large values of buoyancy parameter $\xi=10$ and both values of $A$ which may be a direct result to the velocity overshoot of $f^{\prime}(\eta)$ near the sheet for $\xi=10$.

Fig. 3 shows that for both values of the unsteadiness parameter $A$ the effect of buoyancy parameter $\xi$ is to decrease the temperature $\theta(\eta)$ in the case of assisting flow and increases it in the opposing flow. Also, it is clear from Fig. 3 that the thermal boundary layer thickness decreases as $\xi$ increases. In addition, the effect of buoyancy parameter $\xi$ demonstrates a more pronounced influence on the temperature distribution of a steady flow $(A=0)$ than that of an unsteady one $(A=0.4)$. For given $\xi$, further, it is noticed from Fig. 3 that the effect of $A$ on the temperature $\theta(\eta)$ is found to be more noticeable in the case of opposing flow than that of the assisting flow.

Representative axial velocity, angular velocity and temperature profiles in the case of assisting and opposing flows and various values of the micropolar parameter $\Delta$ are presented in Figs. 4-6. It is found for both positive (assisting flow) and negative (opposing flow) $\xi$ that the axial velocity $f^{\prime}(\eta)$ and angular velocity $h(\eta)$ increase while the temperature $\theta(\eta)$ decreases with an increase in the micropolar parameter $\Delta$ but the effect of $\Delta$ on the velocity and temperature fields is more pronounced in the case of opposing flow. When $\Delta=\mathrm{O}$ (Newtonian fluid), and as $\Delta$ increases, the angular velocity is greatly induced. Further, the micropolar parameter $\Delta$ demonstrates a more pronounced influence on the axial and angular velocities $f^{\prime}(\eta)$ and $h(\eta)$ respectively, than that on the temperature $\theta(\eta)$. Moreover, it is seen from Figs. 4 and 5 that the smaller the $\Delta$, the thinner the momentum and angular momentum boundary layer thickness while the opposite trend is true for the thermal boundary layer as obvious from Fig. 6 .

Figs. 7-9 are the plot of the velocity, microrotation and temperature distribution with $\eta$ for various values of Eckert number $E c$ in the case of assisting and opposing flows. It is known that the viscous dissipation produces heat due to drag between the fluid particles and this extra heat causes an increase of the initial fluid temperature (see Fig. 9). This increase of temperature causes an increase of the buoyant force. Also, there is a continuous interaction between the viscous heating and the buoyant force. This mechanism produces different results in the assisting (upward) and opposing (downward) flow. In the assisting (opposing) flow, the increase in the values of positive (negative) $E c$ will increase the buoyant force in the upward (downward) direction which results in an increase in the fluid velocity as shown in Fig. 7. The positive $(E c>0)$ and negative $(E c<0)$ Eckert numbers assists the upward ( $\xi>0$ and hence $\mathrm{Ec}>0)$ and downward $(\xi<0$ and hence $E c<0)$ flow, respectively as shown in Fig. 7. It is noted from Fig. 8 that the angular velocity $h(\eta)$ first decreases near the sheet surface where $0 \leq \eta<\eta_{0}$ where $\eta_{0} \cong 1.75$ in the case of assisting flow and $\eta \cong 1.8$ in the case of opposing flow but the situation is completely reversed in the other part of the boundary layer where $\eta>\eta_{0}$.

According to the definition of Eckert number, a positive $E c$ corresponds to fluid heating (heat is being supplied across the walls into the fluid) case $\left(T_{w}>T_{\infty}\right)$ so that the fluid is being heated whereas a negative $E c$ means that the fluid is being cooled. From Fig. 9 it is seen that the dimensionless temperature increases when the fluid is being heated $(E c>0)$ but decrease when the fluid is being cooled $(E c<0)$. For $E c<0$ the dimensionless fluid temperature $\theta$ decreases monotonically with $\eta$, from unity at the wall towards its free- 
stream value. It is noted from the definition of $\theta$ that this behaviour implies the monotonous decrease in the actual fluid temperature in the horizontal direction from the sheet temperature $T_{w}$ to the free-stream temperature. On the other hand, for $E c<0$ (i.e. $T_{w}<T_{\infty}$ ) the dimensionless fluid temperature $\theta$ decreases with $\eta$ rapidly at first, arriving at a negative minimum value, for $E c=-4$ and then increases more gradually to its free surface value. Correspondingly, the actual fluid temperature in the horizontal direction increases at first from the surface temperature $T_{w}$ to a maximum value and then decrease to its free-stream value. It should be noted that for the fluid cooling case $(E c<0)$ a negative $\theta$ indicates the excess of actual fluid temperature $T$ over that at the plate because of the viscous dissipation effect.

The axial velocity, angular velocity and temperature profiles in the case of assisting and opposing flows and various values of the radiation parameter $R$ are presented in Figs. 10-12. It is found that for both the axial velocity $f^{\prime}(\eta)$ and angular velocity $h(\eta)$ increase in case of positive $\xi$ (assisting flow) while decrease in case of negative $\xi$ (opposing flow) and the temperature $\theta(\eta)$ decreases with an increase in the radiation parameter $R$ but the effect of $R$ on the velocity and temperature fields is more pronounced in the case of opposing flow.

The axial velocity, angular velocity and temperature profiles in the case of assisting and opposing flows and various values of the heat generation parameter $Q$ are presented in Figs. 13-15. It is observed that for both the axial velocity $f^{\prime}(\eta)$ and angular velocity $h(\eta)$ increase in case of positive $\xi$ (assisting flow) while decrease in case of negative $\xi$ (opposing flow) and the temperature $\theta(\eta)$ decreases with an increase in the heat generation parameter $Q$ but the effect of $Q$ on the velocity and temperature fields is more pronounced in the case of opposing flow.

Typical variations of the local skin friction coefficient in terms of $f^{\prime \prime}(0)$, wall couple stress in terms of $h^{\prime}(0)$ and the heat transfer result in terms of the dimensionless wall temperature gradient $-\theta^{\prime}(0)$ as a function of the unsteadiness parameter $A$ for two representative material parameters, $\Delta=0$ (Newtonian fluid) and $\Delta=3$ (micropolar fluid) and various values of the buoyancy parameter $\xi$ are presented in Figs. 16-18. As compared to the case of forced convection flow $(\xi=0)$,

Figs. 16 and 18 demonstrate that for given $A$ and $\Delta$ the local skin friction coefficient $f^{\prime \prime}(0)$ and the local Nusselt number $-\theta^{\prime}(0)$ increase for positive buoyant force $(\xi=0)$ and decrease for negative buoyant force $(\xi<0)$ while the opposite behavior is true in the case of couple stress $h^{\prime}(0)$ as clear from Fig. 17. In addition, the effect of buoyancy parameter $\xi$ on $f^{\prime \prime}(0)$ and $-\theta^{\prime}(0)$ of a Newtonian fluid $(\Delta=0)$ is more pronounced than that of a micropolar fluid $(\Delta=3)$. From Fig. 16 it is noted that positive skin friction coefficients are obtained for $\xi=10$ which are due to the velocity overshoot as one can see from Fig. 4. It is also observed for all $A$ that the local skin friction coefficient $f^{\prime \prime}(0)$ increases with $\Delta$ for $\xi=-0.5$ (opposing flow), $\xi=0$ (forced convection flow) and $\xi=1$ (mixed convection flow) but the opposite behaviour is obtained for $\xi=10$ (free convection flow). For given $\xi$ and $\Delta$, Fig. 16 reveals that the local skin friction coefficient $f^{\prime \prime}(0)$ decreases as the unsteadiness parameter $A$ increases.

Fig. 17 reveals that for a given $A$ the couple stress $h^{\prime}(0)$ increases significantly with $\Delta$ for negative and lower values of the buoyancy parameter $(\xi=-0.5,0,1)$ but the opposite trend is noticed at larger values of $\xi(\xi=10)$. These behaviours are consistent with the results of the dimensionless angular velocity profiles shown in Fig. 17. Further, for given $\Delta$, the effect of the unsteadiness parameter $A$ on the couple stress $h^{\prime}(0)$ is opposite to that of $\Delta$. Also, the couple stress $h^{\prime}(0)$ is greatly decreased as $\xi$ is increased for both $\Delta$ values but this behaviour is found to be more noticeable at larger values of $\Delta$. For given $\xi$ and $\Delta$, Fig. 18 reveals that the heat transfer is greatly enhanced as the unsteadiness parameter $A$ is increased.

Also, the influence of the unsteadiness parameter $A$ on the heat transfer coefficient $-\theta^{\prime}(0)$ is seen to be more noticeable for lower values of $A$. Further, for given $A$ it is noticed from Fig. 18 that the effect of $\Delta$ is to increase the local transfer coefficient $-\theta^{\prime}(0)$ significantly for opposing flow $(\xi=-0.5)$ and forced 
convection regime $(\xi=0)$ and this effect is reduced for mixed convection regime $(\xi=1)$ but as n increases more $(\xi=10)$, the relation between $-\theta^{\prime}(0)$ and $\Delta$ explained earlier is switched. In other words the micropolar fluid is better suited for effective cooling of the unsteady stretching sheet only when the flow is forced convective. From Fig. 18 it is clear that the heat transfer coefficient increases with $\xi$ for all values of $A$ and both values of $\Delta$ but this trend is seen to be more significant in the case of Newtonian fluid $(\Delta=0)$.

Variations of the local skin friction coefficient $f^{\prime \prime}(0)$, wall couple stress $h^{\prime}(0)$ and the local Nusselt number $-\theta^{\prime}(0)$ as a function of the heat generation parameter $Q$ for various values of the buoyancy parameter $\xi$ and radiation parameter $R$ are presented in Figs. 19-21. It is clear from Fig. 19 that for fixed $Q$ the skin friction coefficient increases as $R$ increases for the assisting flow cases and the skin friction coefficient decrease for the opposing flow case. In addition, the effect of radiation on $f^{\prime \prime}(0)$ is more pronounced for lower values of $Q$. It is also observed that the local skin friction coefficient $f^{\prime \prime}(0)$ of buoyancy assisting flow is higher than that of buoyancy opposing flow for all values of $Q$ and $R$. The effect of radiation on the wall couple stress $h^{\prime}(0)$ is completely opposite to that on the local skin friction coefficient $f^{\prime \prime}(0)$ as obvious from Fig. 20. Further, radiation demonstrates a more pronounced influence on the wall couple stress $h^{\prime}(0)$ in the opposing flow than that of assisting flow. It is clear from Fig. 21 that for fixed $Q$ the local Nusselt number decreases as $R$ increases for both opposing and assisting flow cases. It is also observed that the local Nusselt number $-\theta^{\prime}(0)$ of buoyancy assisting flow is higher than that of buoyancy opposing flow for all values of $Q$ and $R$.

For validation of the numerical method used in this study, results for heat transfer rate $-\theta^{\prime}(0)$ of a Newtonian fluid $(\Delta=0)$ were compared with those of Grubka and Bobba [26] for various values of $\operatorname{Pr}$ in the absence of viscous dissipation $(E c=0)$, radiation $(R=0)$ and heat generation $(Q=0)$. The quantitative comparison is shown in table 1 and it is found to be in excellent agreement.

\section{Conclusions}

In the present prater, the unsteady mixed convection flow of a viscous incompressible electrically conducting micropolar fluid on a vertical and impermeable stretching surface with viscous dissipation by taking radiation and heat generation into account, are analyzed. The governing equations are approximated to a system of non-linear ordinary differential equations by similarity transformation. Numerical calculations are carried out for various values of the dimensionless parameters of the problem. It has been found that

1. The velocity and angular velocity increases as well as the temperature decreases with an increase in the material parameter in both assisting and opposing flows.

2. The heat generation and viscous dissipation enhances the velocity, angular velocity and temperature in both assisting and opposing flows.

3. The unsteadiness parameter enhances the couple wall stress, heat transfer rates.

4. Radiation and heat generation reduces the heat transfer rate for both assisting and opposing flows.

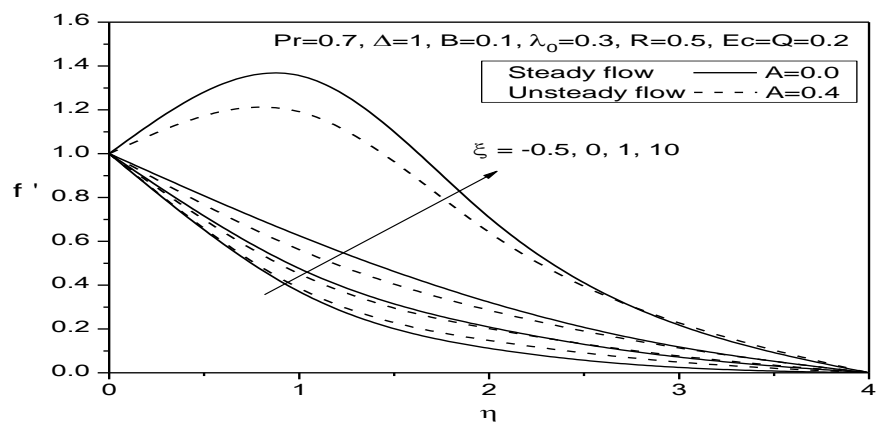

Fig.1 Velocity for different values of $\xi$ and $A$ 


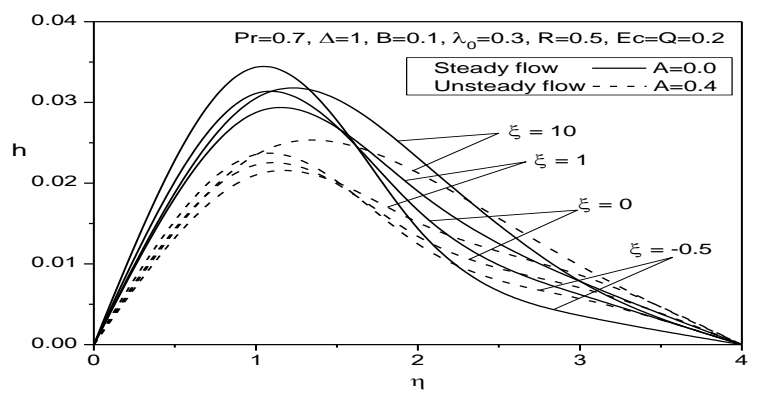

Fig.2 Angular velocity for different values of $\xi$ and $A$

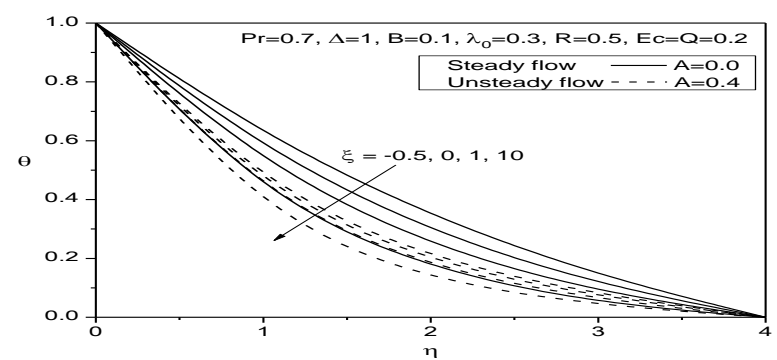

Fig.3 Temperature for different values of $\xi$ and $A$

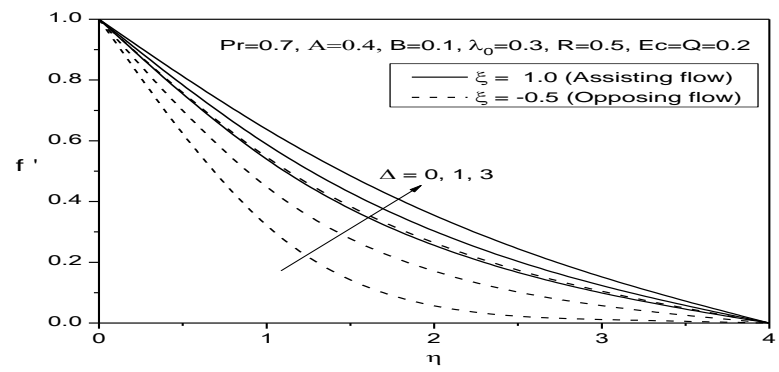

Fig.4 Velocity for different values of $\Delta$ and $\xi$

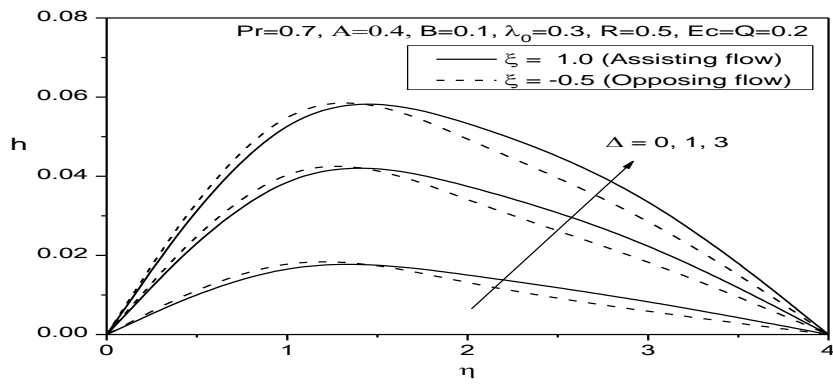

Fig.5 Angular velocity for different values of $\Delta$ and $\xi$

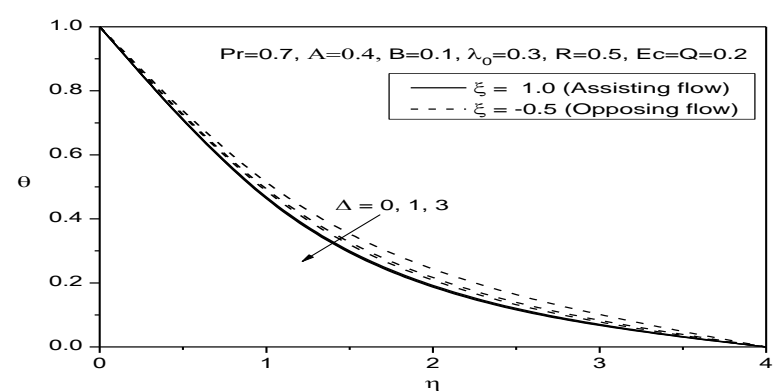

Fig.6 Temperature for different values of $\Delta$ and $\xi$ 


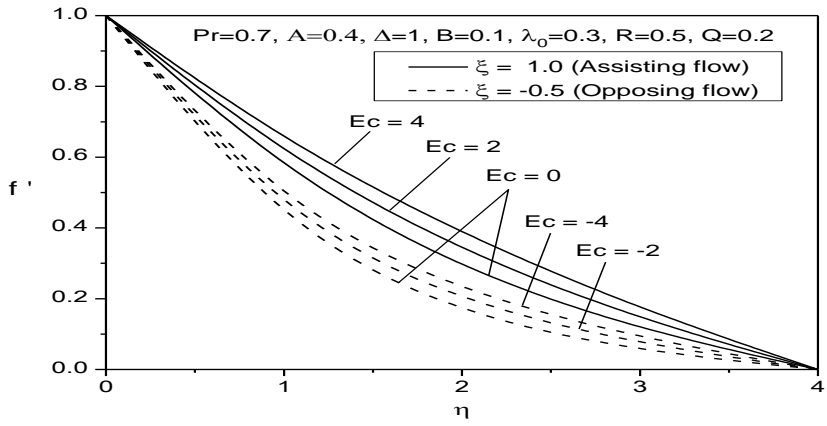

Fig.7 Velocity for different values of $E c$ and $\xi$

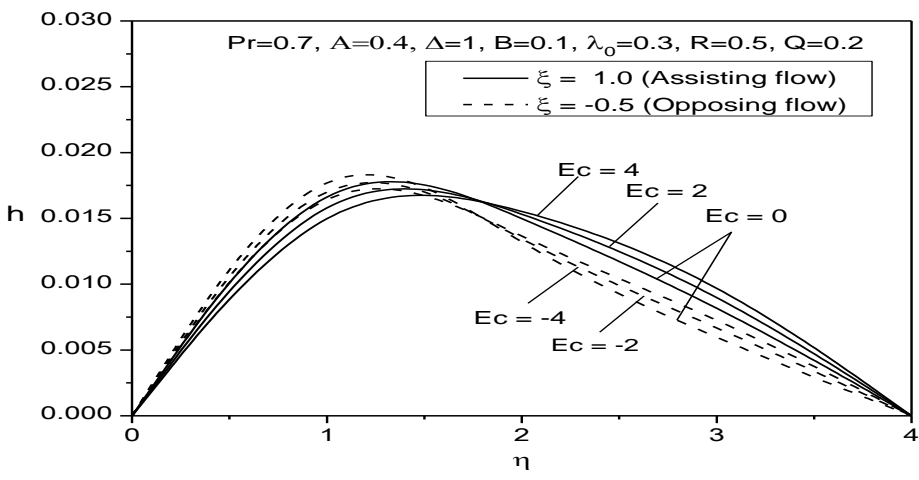

Fig.8 Angular velocity for different values of $E c$ and $\xi$

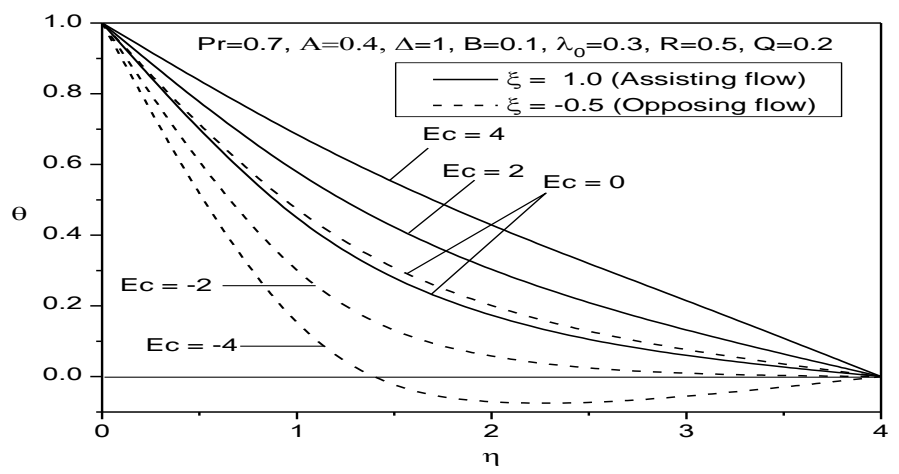

Fig.9 Temperature for different values of $E c$ and $\xi$

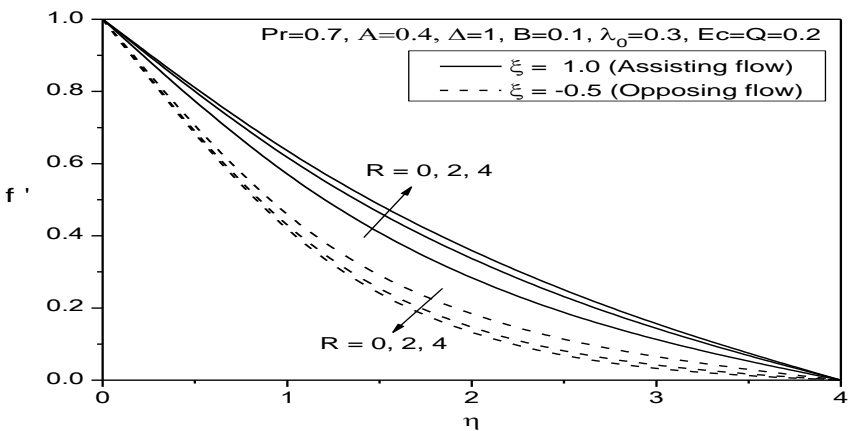

Fig.10 Velocity for different values of $R$ and $\xi$ 


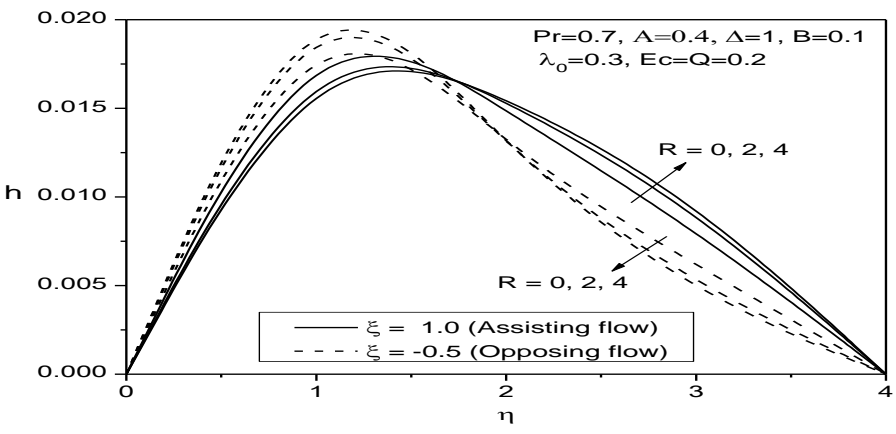

Fig.11 Angular velocity for different values of $R$ and $\xi$

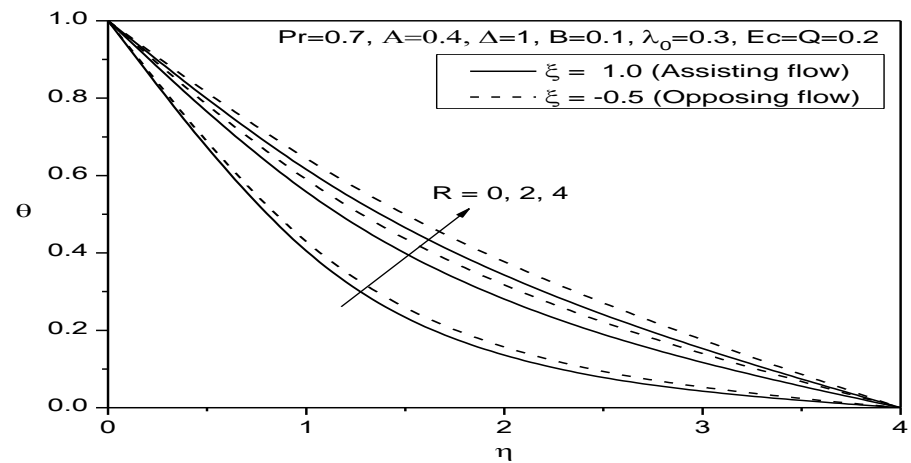

Fig.12 Temperature for different values of $R$ and $\xi$

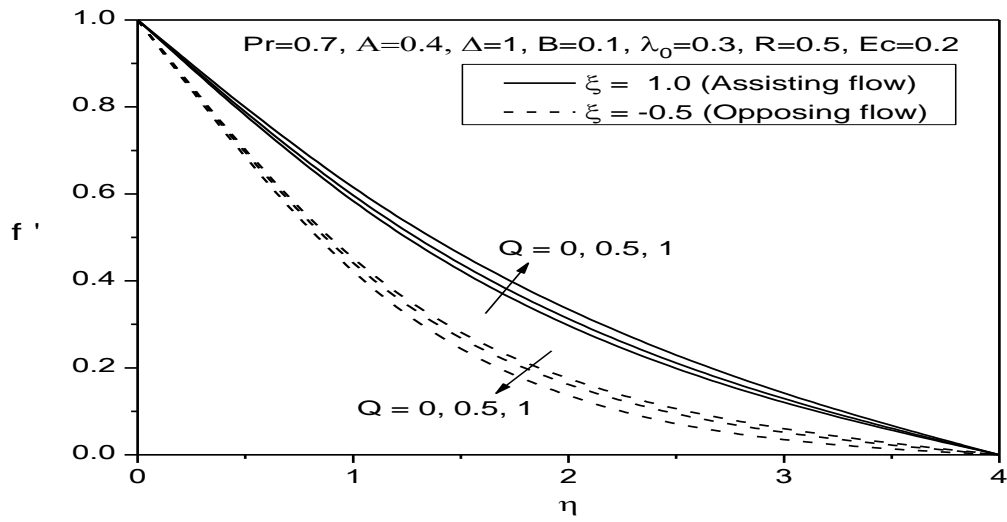

Fig.13 Velocity for different values of $Q$ and $\xi$

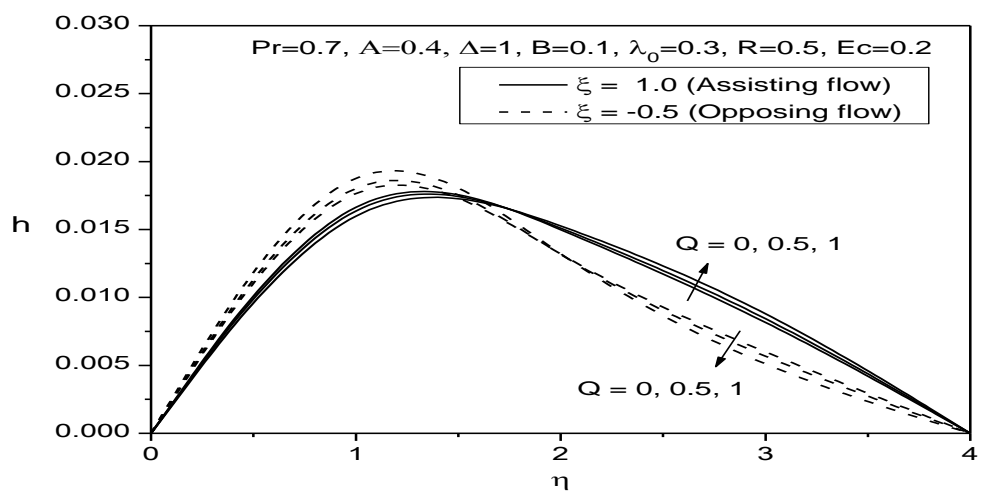

Fig.14 Angular velocity for different values of $Q$ and $\xi$ 


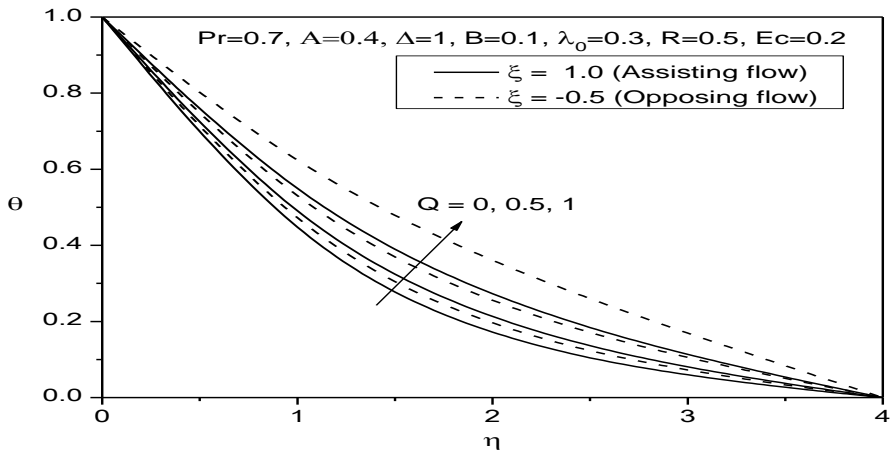

Fig.15 Temperature for different values of $Q$ and $\xi$

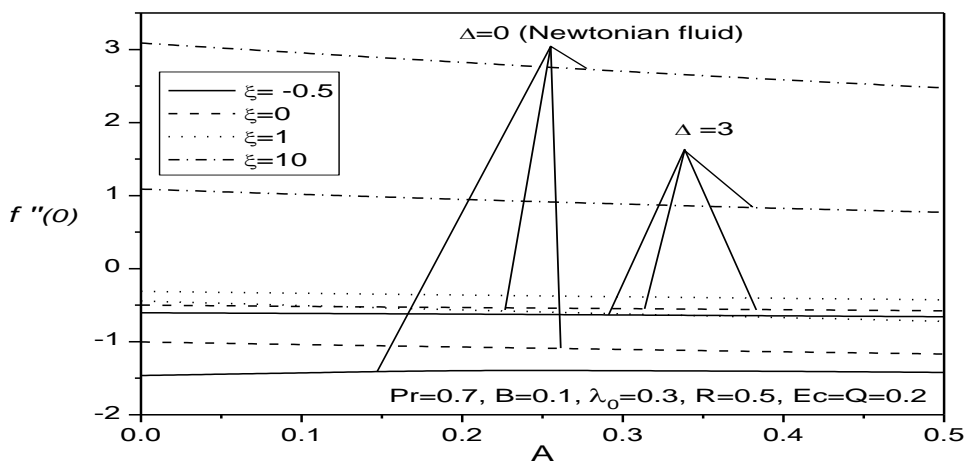

Fig.16 skin friction $f^{\prime \prime}(0)$ vs. $\boldsymbol{A}$ for different values of $\Delta$ and $\xi$

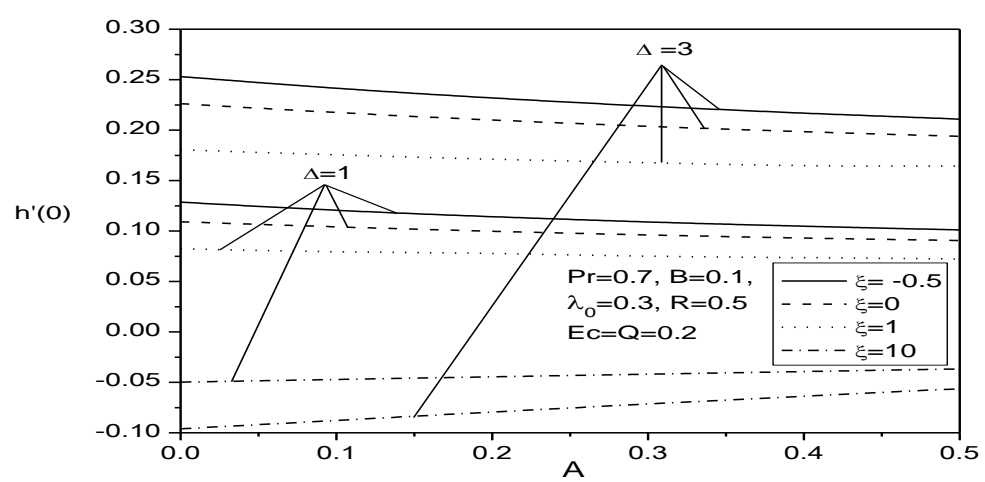

Fig.17 Wall couple stress $h^{\prime}(0)$ vs. $A$ for different values of $\Delta$ and $\xi$

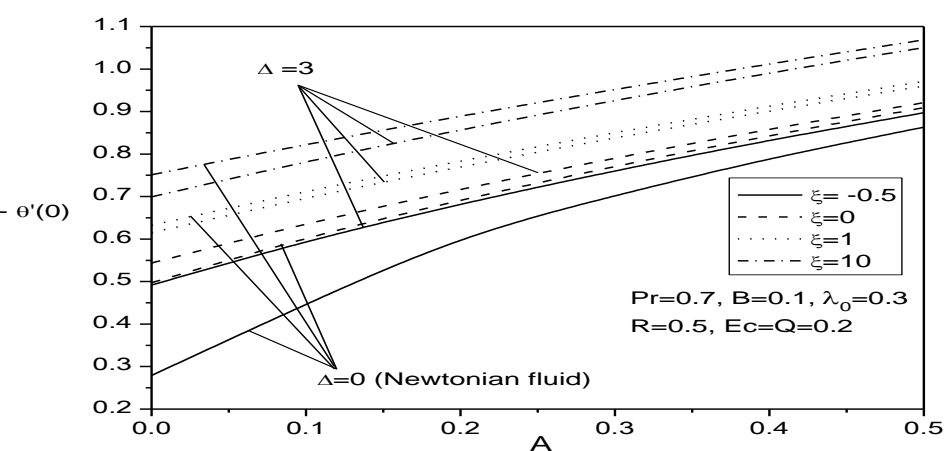

Fig.18 Local Nusselt number $-\theta^{\prime}(0)$ vs. $A$ for different values of $\Delta$ and $\xi$ 


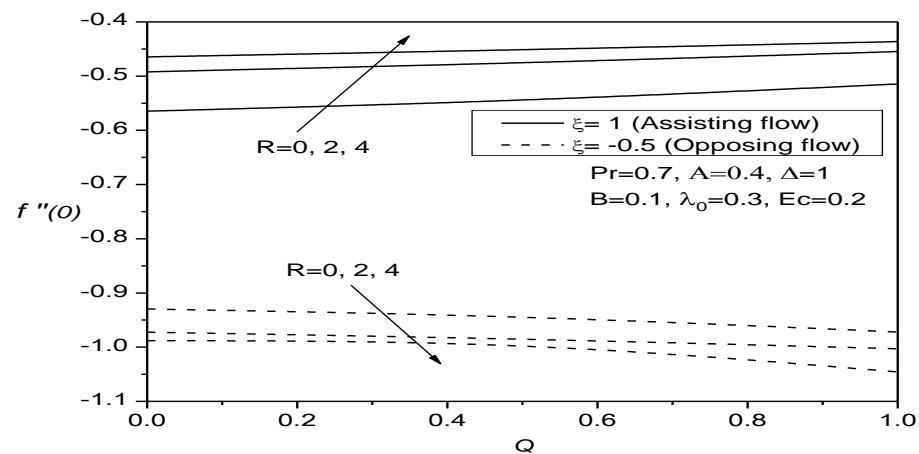

Fig.19 skin friction $f "(0)$ vs. $Q$ for different values of $R$ and $\xi$

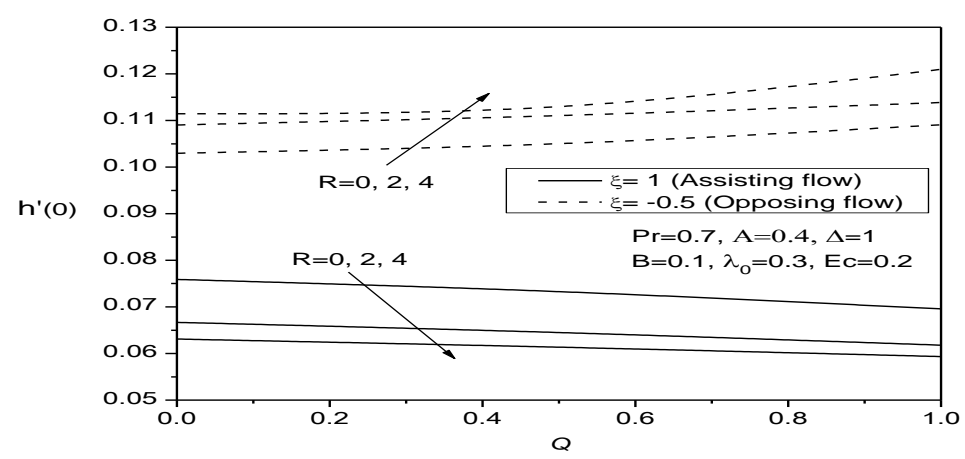

Fig.20 Wall couple stress $h^{\prime}(0)$ vs. $Q$ for different values of $R$ and $\xi$

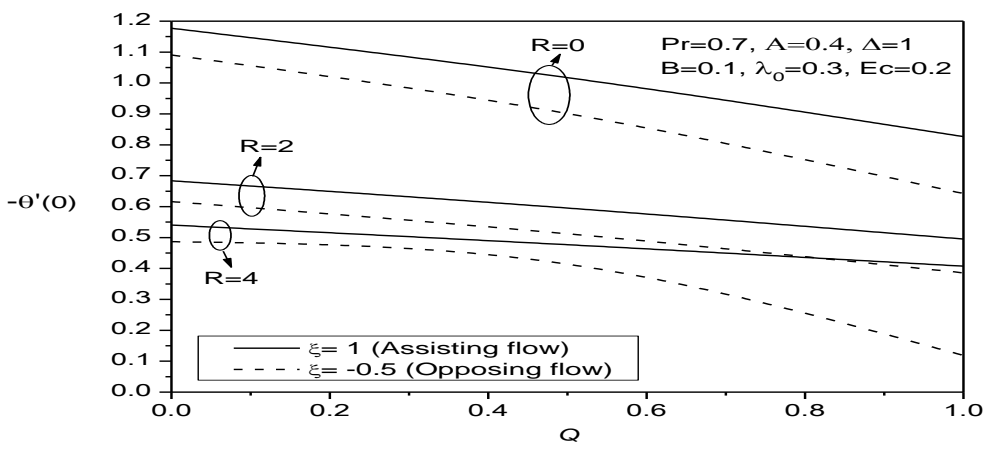

Fig.21 Local Nusselt number $-\theta^{\prime}(0)$ vs. $Q$ for different values of $R$ and $\xi$

Table 1 Comparison of the values of $-\theta^{\prime}(0)$ for $\Delta=E c=A=\xi=R=Q=0$ and various values of Pr with

\begin{tabular}{|c|c|c|}
\multicolumn{1}{c|}{ previously published data. } \\
\hline Pr & \multicolumn{2}{|c|}{$-\theta^{\prime}(0)$} \\
\cline { 2 - 3 } & Ref. [26] & Present study \\
\hline 0.72 & 0.8006 & 0.808731 \\
1.0 & 1.0000 & 1.000000 \\
3.0 & 1.9237 & 1.92368 \\
10.0 & 3.7207 & 3.72067 \\
100 & 12.294 & 12.2904 \\
\hline
\end{tabular}




\section{References}

[1]. Eringen, A.C., (1960), Theory of micropolar fluids, J. Math. Mech., Vol. 6, pp. 1-18.

[2]. Eringen, A.C., (1972), Theory of thermomicropolar fluids, Math. Anal. Appl. J., Vol. 38, pp.481-496.

[3]. Willson, A.J., (1970), Boundary-layer in micropolar liquids, Proc. Cambridge Philos. Soc., Vol. 67, pp.469-476.

[4]. Bergholz, R.F., (1980), Natural convection of a heat generating fluid in a closed cavity, ASME J. Heat Transfer, Vol.102, pp.242247.

[5]. Chandra Shekar, B., Vasseur, P., Robillard, L., Nguyen, T.H., (1984), Natural convection in a heat generating fluid bounded by two horizontal concentric cylinders, Can. J. Chem. Eng., Vol. 62, pp.482-489.

[6]. Peddiesen, J., McNitt, R.P., (1970), Boundary layer theory for micropolar fluids, Recent Adv. Eng., Vol. 5, pp.405-426.

[7]. Lukaszewicz, G., (1999), Micropolar Fluids: Theory and Applications, Birkh a“ user, Boston.

[8]. Eringen, A.C., (2001), Microcontinuum Field Theories. II: Fluent Media, Springer, NewYork.

[9]. Postelnicu, A., Grosan, T., and Pop, I., (2001), The effect of variable viscosity on forced convection over a horizontal flat plate in a porous medium with internal heat generation, Mechanics Research Communications, vol.28, no.3, pp. 331-337.

[10]. Molla, M.M., Hossain, M.A., and Yao, L.S., (2004), Natural convection flow along a vertical wavy surface with uniform surface temperature in presence of heat generation/absorption, Int. J. Thermal Sci., Vol.43, pp.157-163.

[11]. Samad, M.A., and Mohebujjaman, M., (2009), MHD heat and mass transfer free convection flow along a vertical stretching sheet in presence of magnetic field with heat generation, Research J. of Applied Sci., Eng. and Tech., Vol.1(3), pp.98-106.

[12]. Alam, M. S., Rahman, M. M. and Samad, M. A., (2006), Numerical study of the combined free-forced convection and Mass transfer flow past a vertical porous plate in a porous medium with heat generation and thermal diffusion, Nonlinear Analysis: Modeling and Control,vol.11, no. 4, pp. 331-343.

[13]. Mohammed Abdur Rahman, Alim, M. A. and Jahurul Islam, Md., (2013), Thermophoresis Effect on MHD Forced Convection on a Fluid over a Continuous Linear Stretching Sheet in Presence of Heat Generation and Power-Law Wall Temperature, Annals of Pure and Applied Mathematics, Vol. 4, No.2, pp.192-204.

[14]. Vajravelu, K., Hadjinicalaou, A., (1993), Heat transfer in a viscous fluid over a stretching sheet with viscous dissipation and internal heat generation. Int. Comm. Heat Mass Trans. 20, 417-430.

[15]. Gebharat, B., (1962), Effects of viscous dissipation in natural convection, J. Fluid Mech., Vol. 14, pp.225-232.

[16]. Gebharat, B., and Mollendorf, J., (1969), Viscous dissipation in external natural convection flows, J. Fluid. Mech.,Vol.38, pp.97107.

[17]. Mohamed Abd El-Aziz, (2013), Mixed convection flow of a micropolar fluid from an unsteady stretching surface with viscous dissipation, Journal of the Egyptian Mathematical Society, Vol. 21, pp.385-394.

[18]. Ghaly, A.Y.: Radiation effect on a certain MHD free convection flow. Chasos, Solitons Fractal 13, 1843-1850 (2002)

[19]. Rapits, A., Massalas, C.V.: Magnetohydrodynamic flow past a plate by the presence of radiation. Heat and Mass Transf. 34, 107109 (1998)

[20]. El-Aziz, M.A.: Radiation effect on the flow and heat transfer over an unsteady stretching surface. Int. Commu. Heat and Mass Transf. 36, 521-524 (2009)

[21]. Sattar, M.A., Alam, M.M.: Thermal diffusion as well as transpiration effects on MHD free convection and mass transfer flow past an accelerated vertical porous plate. Indian J. of Pure App. Math. 25, 679-688 (1994)

[22]. Na, T.Y., Pop, I.: Unsteady flow past a stretching sheet. Mech. Res. Comm. 23, 413-422 (1996)

[23]. Singh, P., Jangid, A., Tomer, N.S., Sinha, D.: Effects of thermal radiation and magnetic field on unsteady stretching permeable sheet in presence of free stream velocity. Int. J. Info. and Math. Sci. 6, 3 (2010)

[24]. Md Shakhaoath Khan, Ifsana Karim, Lasker Ershad Ali and Ariful Islam, (2012), Unsteady MHD free convection boundary-layer flow of a nanofluid along a stretching sheet with thermal radiation and viscous dissipation effects, International Nano Letters, 2:24, pp.1-9.

[25]. Jain, M.K., Iyengar, S.R.K. and Jain, R.K., (1985), Numerical Methods for Scientific and Engineering Computation, Wiley Eastern Ltd., New Delhi, India.

[26]. Grubka, L.J., and Bobba, K.M., (1985), Heat transfer characteristics of a continuous stretching surface with variable temperature, ASME J. Heat Transfer, Vol.107, pp.248-250. 